\title{
Réseaux sociaux et défi démocratique : l'exemple du cyberespace camerounais
}

Social networks and democratic challenge: the example of the Cameroonian

\section{Aboubakar Sidi Njutapwoui et Jean Pierre Fewou Ngouloure}

\section{(2) OpenEdition}

12 Journals

Édition électronique

URL : http://journals.openedition.org/ctd/1772

DOI : $10.4000 /$ ctd. 1772

ISSN : 2491-1437

Éditeur

Chaire Unesco Pratiques émergentes en technologies et communication pour le développement

Édition imprimée

Date de publication : 12 novembre 2015

\section{Référence électronique}

Aboubakar Sidi Njutapwoui et Jean Pierre Fewou Ngouloure, «Réseaux sociaux et défı démocratique:

l'exemple du cyberespace camerounais ", Communication, technologies et développement [En ligne], 2 ।

2015, mis en ligne le 12 juin 2019, consulté le 22 juillet 2020. URL : http://journals.openedition.org/

ctd/1772 ; DOI : https://doi.org/10.4000/ctd.1772

Ce document a été généré automatiquement le 22 juillet 2020.

Communication, technologies et développement 


\title{
Réseaux sociaux et défi démocratique : l'exemple du cyberespace camerounais
}

Social networks and democratic challenge : the example of the Cameroonian

\author{
Aboubakar Sidi Njutapwoui et Jean Pierre Fewou Ngouloure
}

\section{Introduction}

1 Longtemps soumis aux dures réalités du parti unique marquées par l'absence de liberté de parole et de pensée, le citoyen ordinaire camerounais a rarement été acteur de son propre destin en matière de démocratie. Même avec l'ouverture à la pluralité politique opérée dans les années 90, qui a vu la multiplication des partis politiques et un desserrement de l'étreinte de la censure et de l'oppression par le pouvoir en place, celui-ci a toujours réussi à maintenir la pression pour étouffer toute tentative d'insurrection de la part des sans-voix. C'est pourquoi les médias publics ont toujours dominé ceux qui relèvent de la sphère privée, jouissant pour cela de financement suffisant et conséquent, d'où une grande couverture à l'échelle nationale, voire internationale pour pouvoir toucher un public aussi large que possible. En revanche depuis l'avènement des réseaux sociaux, l'on note de plus en plus un renversement de la hiérarchie des valeurs où même celles et ceux qu'on pouvait considérer jusque-là comme des laissés pour compte prennent une sorte de revanche en s'appropriant le cyberespace pour promouvoir une certaine culture démocratique.

2 En ce sens, les réseaux sociaux sont en train de consacrer une nouvelle catégorie d'individus capables de jouer le rôle majeur dans la sphère publique, bien que dans un registre populaire, sur des sujets relevant aussi bien des préoccupations politique, économique et sociale. Internet serait désormais dans cette optique l'outil structurant d'une démocratie participative et délibérative (Trippi, 2008). Cela présagerait du coup de la naissance d'un nouvel espace politique (Castells, 2001) et de la reconfiguration de l'espace public dans un pays où l'État monopolisait jusque-là l'expression politique, au 
point de constituer le cinquième pouvoir (Grallet et al, 2006). L'objet de notre étude est ainsi de montrer ce qu'ont changé véritablement les réseaux sociaux en termes d'(ré)appropriation démocratique au Cameroun. Nous chercherons aussi à savoir quelles peuvent être les incidences d'une parole finalement non contrôlée et échappant parfois aux règles de l'éthique la plus élémentaire. En effet, des travaux tendent à démontrer que les usages d'Internet participeraient d'un autre point de vue au renforcement et à la (re)production d'une hiérarchie de pouvoir (Freitag, 2003).

Evgeny Morozov (2014) considère par ailleurs que si les médias numériques peuvent jouer un rôle dans l'organisation de mouvements sociaux, ils sont incapables de fédérer des alliances durables, ou même de protéger les militants une fois que le pouvoir s'engage à utiliser les mêmes outils pour sévir contre toute dissidence. Et pour répondre à toutes ces interrogations, nous allons fonder notre analyse sur deux sites web d'information camerounais: cameroun-info.net ${ }^{1}$ et Camer be ${ }^{2}$. Ces deux sites permettent à tout contributeur d'interagir en ligne au travers des espaces de discussion ouverts à cet effet. Il s'agira d'étudier quelques commentaires d'internautes triés en fonction de trois principaux critères: leur lien avec le registre et les sujets démocratiques; tout indice démontrant directement ou indirectement l'identité de l'internaute comme relevant d'une minorité (tribale, sociale, politique, etc.); le caractère spécifique du post retenu (incidence par exemple sur le cadre juridique). Parlant enfin du cadre méthodologique, nous nous appuierons sur une analyse à la fois systémique, en étudiant le rôle des médias sociaux en tant qu'organisation, c'est-à-dire comme un champ de pouvoir; et sociologique, à travers le concept de " constructivisme social ", pour découvrir la manière dont la réalité sociale et les phénomènes sociaux sont mis en branle dans les médias sociaux.

\section{Approche méthodologique}

4 Analyser un corpus numérique n'est jamais du tout repos, du fait de son caractère fluctuant, hétérogène, voire hétéroclite. Ainsi, les données d'un corpus numérique peuvent à tout moment être changées, modulées, supprimées, censées par des internautes ou par les modérateurs de sites web, dans des situations de dérapage, ce qui est de nature à poser d'énormes difficultés dans leur collecte. C'est le même constat que l'on peut faire lorsqu'on parle de « cyberespace » pour délimiter un cadre géographique bien précis, quand on sait par exemple qu'Internet a la vocation première de permettre l'interconnexion mondiale entre les humains, les machines, les objets ou toutes les données liées au domaine informationnel ou communicationnel, indépendamment de leur territoire. Le postulat d'un « cyberespace camerounais » que nous posons dans la présente étude semble dès lors relever d'une dimension presque symbolique, à défaut d'être utopique, le caractère opaque qui fonde l'espace web en général ne permettant pas toujours de délimiter les contours d'un univers qui se veut sans frontière.

5 Cela dit, au risque de vouloir constituer un échantillon faussement représentatif, la meilleure solution pour laquelle nous avons opté dans cette réflexion consiste plutôt à étudier dans le détail quelques occurrences, qui sont des interactions des internautes qui se présentent comme étant victimes d'un système étatique où dominent les injustices sociales. Notre observation, qui se fonde davantage sur une démarche de type qualitatif, s'appuie donc sur un corpus constitué de quelques commentaires publiés sur les deux sites camerounais en ligne. Il s'agit précisément des interactions langagières 
dont le topique, principalement sociopolitique, concerne les minorités dans leur dynamique communicationnelle.

6 Le choix de l'approche systémique pour conduire cette étude s'explique d'une part par le fait que les espaces de discussion et d'échanges peuvent être considérés selon Michel Crozier et Michel Erhard (1981) comme des organisations qui constituent pour les acteurs un champ d'investissement stratégique. L'organisation évoque avant tout un ensemble de réseaux complexes, mais parfaitement agencés. L'analyse systémique permet dans ces conditions de voir que les acteurs ne s'engagent dans ces relations virtuelles que dans la perspective de trouver dans ces espaces des "enjeux" suffisamment «pertinents» au regard de leurs "atouts» et de leurs objectifs. En d'autres termes, leur participation à l'organisation est conditionnée par des intérêts parfois précis, à savoir une certaine quête de visibilité médiatique. Bien plus, on peut constater pour ce qui est de notre espace d'études que ce sont des réseaux d'individus parfois liés entre eux par des considérations culturelles communes. C'est à ce niveau que le rôle des réseaux et médias sociaux, en tant qu'organisation, c'est-à-dire un champ de pouvoir, devient encore plus important. L'analyse des occurrences sélectionnées permettra en clair de voir quels sont les critères qui définissent la " pertinence » de ces ressources et leur caractère plus ou moins mobilisable.

7 D'autre part, parlant du constructivisme social, nous partons de l'hypothèse que les acteurs en proie à la marginalisation sociale impliqués dans la dynamique communicationnelle dans le cyberespace camerounais cherchent à construire une sociologie dont le langage doit empêcher toute légitimation de l'ordre existant. Il s'agit alors de questionner tout le potentiel de structuration de l'esprit humain que les groupes dominés peuvent mobiliser dans les plateformes d'échanges numériques pour déconstruire et délégitimer leur domination par le pouvoir étatique. Bref, le constructivisme social fait percevoir comment les minorités se saisissent de la liberté d'expression, construisent symboliquement le débat en faveur de leur cause dans le but d'influencer l'opinion publique nationale et internationale.

\section{Internet au Cameroun : un nouvel espace de liberté ?}

Selon un rapport rédigé par trois organisations non gouvernementales internationales et remis à l'Organisation des Nations Unies (ONU), la liberté d'expression serait en danger au Cameroun. ${ }^{3}$ Il s'agit de PEN International, Committee to Protect Journalists et Internet sans frontières. Dans l'une des conclusions qu'elles ont formulées, il est clairement indiqué que le Cameroun est un "pays dans lequel être un écrivain ou journaliste, à la fois en ligne et hors ligne, est risqué ». De même ces trois organisations estiment que « de nombreuses lois pénales punissent les écrivains et les journalistes en raison de leur travail, autorisent les détentions provisoires de longue durée dans des prisons surpeuplées, la torture systématique, et favorisent l'inexistence de procès équitable ». Le gouvernement aurait également durci le ton sur la presse en imposant, de manière sélective, aux journaux et publications des licences coûteuses. Il est également établi d'après les investigations de ces trois organisations que les menaces sur la liberté d'expression persistent également en ligne. Elles relèvent ainsi que le Cameroun a bloqué du contenu sur Internet et encourage la privatisation de l'application de sa loi contre la cybercriminalité, sans contrôle judiciaire suffisant. Du coup moins de $5 \%$ des Camerounais auraient accès à Internet (données datant de 2012). 
Ce faible taux de pénétration s'expliquerait en clair par le contrôle rigoureux que l'État camerounais exerce sur l'infrastructure et une réglementation stricte, qui ont pour conséquence des coûts exorbitants pour les fournisseurs d'accès à Internet et les utilisateurs. Aujourd'hui, deux agences sont chargées de gérer et de protéger les télécommunications au Cameroun. Il s'agit de l'Agence de Régulation des Télécommunications (ART) et de l'Agence Nationale des Technologies de l'Information et de la Communication (ANTIC), qui contrôlent les activités de sécurité des réseaux de communications électroniques. Elles disposent des pouvoirs de surveillance, d'investigation, d'injonction, de coercition et de sanction. Ainsi, la publication de tout contenu illicite peut être réprimée par la loi relative à la cybercriminalité et à la cybersécurité4.

Toutefois, malgré ce tableau peu reluisant de la situation du Cameroun en matière de liberté d'expression, il s'organise de plus en plus sur des espaces de discussion en ligne des aspirations à la révolte populaire de la part des internautes qui estiment leurs droits bafoués. La parole s'est donc peu à peu libérée, de nombreuses interventions des usagers du web défiant clairement le régime en place sur le registre de l'invective, voire de l'injure, ce qui est fort difficile, voire pratiquement impossible, au travers des médias traditionnels comme la télévision et la presse écrite nationales. Les réseaux sociaux constituent en somme au Cameroun, malgré quelques réserves, un espace où des usagers peuvent venir exprimer leurs opinions, y compris ceux qui se considèrent comme relevant des couches minoritaires.

\section{La difficile question des minorités}

10 La question des minorités n'est pas un problème spécifiquement camerounais. S'il existe une Sous-commission de la lutte contre les mesures discriminatoires et de la protection des minorités au niveau des Nations-Unies, cela montre bien qu'il s'agit d'un problème ayant une portée mondiale. Cela dit, ce sujet est assez complexe puisqu'il revêt un caractère à la fois démographique, économique, linguistique, intellectuel, social, culturel, confessionnel, etc. D’une manière générale, la difficulté à définir ce qu'est une minorité découle du fait qu'il est souvent difficile de savoir sur la base de quel(s) critère(s) fonder ses choix heuristiques. Certains auteurs proposent de partir d'un point de vue quantitatif. En cela, les minorités seraient un groupe inférieur numériquement par rapport à l'ensemble d'une population donnée :

«Une minorité, dit Norbert Rouland, [...] est un groupe humain marginalisé, se trouvant en position d'infériorité par rapport à la société globale et, en général, à

l'État-nation, dans lesquels elle se trouve incluse ». (Rouland, $1990: 155$ ).

11 D'autres proposent en revanche de situer le point de vue dans une perspective qualitative. Dans cette logique, les minorités seraient pour l'essentiel un groupe en position de non dominance, marginalisée, maltraitée, comme fut par exemple la population noire sous le régime de l'apartheid en Afrique du Sud ou encore des Tutsi au Rwanda lors du génocide de 1993. Notre orientation de recherche s'inscrit dans cette dynamique définitionnelle puisqu'il s'agit pour nous de nous intéresser à des catégories sociales dont l'un des objectifs et de profiter du cyberespace pour se donner des moyens de se faire entendre afin de conjurer à leur manière les injustices qui affectent leur existence. 


\section{Qui sont les minorités au Cameroun?}

12 La question des minorités dans la législation camerounaise reste globalement lacunaire selon Rodrigue Ngando Sandjé (2013) et s'écarterait de la philosophie de la société internationale, $d u$ fait d'un véritable manque de prise en compte des réalités locales. Selon lui, parmi les freins à une meilleure appréhension des enjeux locaux qui relèvent de la problématique des minorités, la question des autochtones serait particulièrement celle qui divise la communauté scientifique camerounaise. En effet, du fait du brassage des populations locales, vecteur des identités métisses, il est souvent difficile d'identifier des prototypes monoethniques. Si être autochtone est lié dans le droit camerounais à l'appartenance physique à un territoire dans une dynamique ancestrale et essentialiste, il n'en demeure pas moins vrai qu'avec les nouvelles réalités liées à la globalisation, cette appartenance peut aussi revêtir des caractéristiques psychologiques et affectives. Il ressort ainsi dans l'une des hypothèses de l'auteur que le droit camerounais des minorités a oublié, voire nié les groupes marginaux. Dans la même logique, Jean Njoya estime, toujours dans le cas du Cameroun, que «la minorité est confinée à une protection virtuelle et aléatoire ». (Njoya, $2011: 25)$. Cela dit, la constitution camerounaise retient surtout le critère ethnique et non linguistique ou religieux pour identifier les groupes minoritaires. Cela peut d'une certaine manière avoir du sens lorsqu'on sait qu'il s'agit d'une société pluraliste où sont parlées plus de 250 langues nationales, avec celles qui ont même une audience régionale. C'est sans doute la raison pour laquelle «la conception, la catégorisation et même le régime du droit des minorités sont faussés au Cameroun » (Ngando Sandjé, op.cit : 152).

Plusieurs auteurs camerounais (politologues, sociologues, juristes, anthropologues, etc.,) se sont tout de même penchés sur les problèmes des minorités anglophones (Konings \& Nyamnjoh, 1997). D'autres travaux ont fait également cas des minorités Mbororo de l'Adamaoua et du Nord-Ouest anglophone (Burnham, 1996 ; Pelican, 2008), ainsi que des minorités Mbo, Pouakam, Tikar et Mbororo de l'ouest-Cameroun (Mouiche, 2010). Si les différentes identifications opérées des minorités accordent la part belle aux critères géographiques et socioculturels, notre orientation se voudra davantage politique et sociale. Pour nous, la notion de minorité aura bel et bien une connotation politique, et il sera question de parler des couches considérées comme marginalisées du fait de leur manque de reconnaissance sociale dans la gestion de la cité. En ce sens, Rodrigue Ngando Sandjé montre par exemple que le critère sexuel est un facteur et un creuset de discrimination au Cameroun. Il s'appuie pour illustrer ses propos sur les élections législatives organisées au Cameroun en 2007, qui a vu l'élection de 23 femmes pour un total de 180 députés, ce qui prouve bien que le poids politique des femmes est faible dans ce pays et qu'elles restent encore installées dans de sortes de ghettos politiques. Il n'existe même pas encore à ce jour de débat sur la parité politique alors qu'il permet d'enrichir "[...] la réflexion sur les principes fondateurs d'une République démocratique ». (Pisier et Varikas, 1997 : 127).

Dans le même ordre d'idées, dans une interview accordée au journal le monde le 26 janvier 2015, Achille Mbembé, l'un des intellectuels camerounais les plus connus, s'offusque du fait que les jeunes soient mis au ban par le pouvoir en place, pour tout ce qui concerne la promotion sociale et politique ${ }^{5}$. On comprend mieux pourquoi dans notre hypothèse directrice, nous considérons les jeunes et les femmes comme étant potentiellement des catégories minoritaires, du point de vue politique et social. C'est en 
effet une des curiosités du Cameroun : être jeune ou femme s'avère comme un lourd fardeau à porter pour des personnes qui veulent gravir des échelons au niveau social, par le travail et l'effort. La gérontocratie et le système local où domine encore le patriarcat sont loin de favoriser l'égalité sociale. Bref, si ces deux catégories sociales ne répondent certes pas aux critères retenus dans les définitions avancées sur les minorités, dans le contexte camerounais, à l'évidence, appartenir à ces deux sphères de la population, à défaut d'avoir de bons réseaux familiaux et relationnels, reste tout de même un handicap.

\section{Un laborieux apprentissage démocratique via le cyberespace}

De nombreuses études articulant le lien entre Internet et démocratie ont déjà été réalisées (Flichy, 2008). Selon cet auteur, Internet joue effectivement un rôle positif dans la dynamique démocratique. Toutefois, il ressort d'autres travaux une portée plutôt négative du web dans la promotion de l'esprit démocratique. Ils semblent ainsi disqualifier de façon durable l'impact positif du débat public en ligne, qui est souvent le siège des guerres d'injures (Dumoulin 2002), où émergent des " monologues interactifs". Même si l'idée d'une communauté d'intérêt en ligne (Lickider et Taylor, 1990) est de plus en plus partagée, il faut néanmoins reconnaître qu'il est souvent difficile pour les internautes de partager des points de vue communs, du fait de la coexistence des identités multiples. Ces points de vue sont d'ailleurs souvent marqués du sceau de la rigidité, chaque usager semblant considérer toute flexibilité de position comme un signe de faiblesse et de renoncement à ses convictions propres. Dans cette intervention d'un internaute camerounais, le débat qui aurait pu rester policé finit par tourner à la fois d'empoigne, suite à l'interview d'une figure locale du cinéma, Bassek Ba Khobio, très connue sur le site Camer be :

«pour ta gouverne et ta culture générale en lieu et place de ton tri-ba-lis-me qui te rend zinzin, Bassek Bakhobio n'est pas bhams, il est bassa. espèce de choigne. t'es tellement tri-ba-le que tu perds la tête, pauvre de toi. $»^{6}$

Rappelons pour éclairer le lecteur que le terme «bhams» est une appellation péjorative de "Bamilikés ", populations souvent considérées à tort ou à raison comme étant sous-représentées du point de vue politique, malgré leur poids considérable dans le domaine économique. Parlant de cette intervention proprement dite, elle pose un problème d'éthique communicationnelle puisque l'internaute fait le choix de l'injure et de l'irrespect de l'autre. Or cette attitude peut être punissable, comme il est prévu dans les lois en vigueur au Cameroun. D'une certaine manière, ces échanges, bien qu'inscrits dans un registre démocratique, au sens de la liberté d'expression, n'en restent moins tributaires d'une forme de dégénérescence de l'espace public, selon les termes même de Jürgen Habermas, situation qui découle d'une difficile "culture de l'intégration" (Habermas, 1993 : 224), qui consiste à trouver des points de consensus sur des intérêts opposés ou sur des sujets où les opinions sont divergentes.

Pire encore, cet autre internaute n'hésite pas à considérer Paul Biya comme un «monstre », tout en voyant son post passer entre les mailles du filet de la modération. En réalité, ce qu'il dénonce avec des mots aussi durs et une emphase aussi dure, c'est plus la longévité de ce président au pouvoir que sa «cruauté » en tant que telle. Le registre est métaphorique, mais sa connotation est ostensiblement hyperbolique. Il cherche en fait à user du pouvoir des mots pour inscrire sa présence sur le web dans un registre conflictuel. C'est une autre façon d'organiser le contre-pouvoir par un usage 
abusif d'un vocabulaire hostile, mais sans véritable vision d'un modèle alternatif, ce qui peut tendre à disqualifier ce type de discours comme légitime :

«"la fin du cauchemard devrait etre le debut d'un cauchemard plus cauchemardeque" le malheur des uns n'est-il pas le bonheur des autres? pour la plus grande majorite des camerounais la disparition quelque soit la manière de ce sinistre monstre sera un grand soulagement ${ }^{7}$

Cela prouve s'il en était besoin que même s'il règne une sorte de terreur au Cameroun, concernant la liberté d'expression, l'espace web constitue tout de même un terrain de répit pour dénoncer l'abus du pouvoir des responsables politiques. Ce qui compte finalement pour cet internaute, ce n'est sans doute pas de vouloir s'engager dans un défi démocratique en faisant preuve de tact communicationnel et de retenue verbale. Il s'agit manifestement d'exprimer publiquement une position et d'exister sur la toile comme citoyen à part entière capable de faire part des préoccupations politiques de son pays en matière de justice sociale. Au regard de ce qui précède et par rapport à l'ensemble de nos hypothèses directrices, nous pouvons dire que les interactions discursives sur le cyberespace camerounais permettent de dresser trois constats majeurs :

- Bien que ce cyberespace offre des espaces de libre expression pour les minorités au Cameroun, leur meilleur usage à des fins sociopolitiques reste encore mitigé, à ce jour.

- L'absence d'une véritable culture politique et numérique par ces minorités, combinée au retard économique dont elles sont les principales victimes, ralentit encore l'émergence d'un vrai modèle de communication maîtrisé et décentralisé. Les pratiques de démocratie ayant cours semblent encore s'inscrire dans le prolongement de celles habituellement observées dans leur quotidien, où la communication rime en quelque sorte avec une certaine anarchie.

- Ces espaces d'échanges et de discussion offrent toutefois à ces minorités des ressources nouvelles de liberté et de communication qui peuvent être mobilisées en faveur de leur intégration. La communication des minorités via le web, même dénuée de véritable stratégie, est donc susceptible d'exercer un effet positif sur leur participation politique, pour plus d'équité et de justice sociales.

19 Force est de constater, en regard de cette dernière hypothèse, que certains frémissements démocratiques, notamment lors des mouvements du printemps arabe, ont pu être observés grâce au rôle crucial des réseaux sociaux, seul moyen de mobiliser les foules en contournant les médias traditionnels largement contrôlés par le pouvoir étatique. Dans le cas de la révolution tunisienne, Younès Boughzala, Inès Bouzid et Jean Moscarola (2012) expliquent à cet effet que c'est par cet outil moderne de communication que les Tunisiens ont réussi à surmonter et à vaincre le démon de la peur pour sortir dans la rue et appeler à la révolte populaire.

\section{En lutte pour une meilleure représentation nationale au niveau politique et social}

Dans le contexte camerounais, certaines catégories s'estiment minoritaires, quant à la distribution des postes de responsabilité au sein de l'appareil étatique. Elles pensent que les Fang-Beti, qui font partie de l'ethnie du président national camerounais, détiennent l'essentiel du pouvoir. La stratégie d'interaction discursive consiste dès lors à créer des micro-espaces publics pour assurer la visibilité à l'échelle numérique, pour les couches sociales désocialisées. En termes de posture discursive, la différence touche davantage au choix des mots, qui peuvent relever des niveaux iréniques et/ou 
polémiques. Dans le cyberespace camerounais, de nombreuses interventions observées peuvent ainsi dans l'un ou l'autre sens :

«Avertissement!!! Que Biya tente de positioner un autre fang-béti boulou au pouvoir, on va pas leur dire. on a été clairet on ne peut pas être plus clair que ça. Just wait and see $»^{8}$

$21 \mathrm{Au}$ nom de ce qu'il est convenu d'appeler «équilibre régional», cet internaute n'accepte pas que le président camerounais puisse céder le pouvoir à un membre de sa communauté ethnique, une fois qu'il consentira à le quitter, d'où ces propos sous fond de menace. Cette mise en garde offensive, en phase avec une sorte de démocratie en action, montre bien qu'il n'appartient pas lui-même à cette ethnie de privilégiés qu'est le peuple beti. Au niveau de la représentation politique au Cameroun, les Beti sont globalement considérés par de nombreux observateurs politiques comme «majoritaires " puisqu'ils occupent la plupart de grands postes de responsabilité. En bref, selon cet internaute, la démocratie ne doit pas reposer sur des considérations ethniques mais sur des critères qui fondent des valeurs où la logique communautariste est vouée aux gémonies. Tel semble ne pas être le cas de cet autre intervenant qui semble, en regard de son propos, appartenir au groupe beti en question. Il n'hésite pas dans cette optique à marteler que le président Paul Biya "pobia " va effectivement s'éterniser au pouvoir, au mépris de toute règle démocratique :

« encore un autre bam's qui veut salir l'image du cameroun.pobia sera eternel allez dire.bande de bamile con $»^{9}$

Cette intervention, qui a sans doute pour but de permettre à l'intervenant d'occuper l'espace médiatique, et d'appeler sans doute à la polémique, vise à fragmenter et à fragiliser le débat public. Il n'y a aucune place ici pour toute médiation où le sentiment national peut au moins de temps en temps prendre le pas sur le sentiment d'injustice, comme peuvent le vivre tous ceux qui s'estiment écartés de la gestion de la cité, du fait de leur ethnie, sexe, etc. En revanche, dans cet autre exemple, l'internaute fait bien la distinction entre démocratie et autocratie, même si l'on reste dans le même registre où domine la violence verbale :
« Démocratie ou tribucratie que voulez-vous? Est-ce que le president du Cameroub est nommé ou élu ? [...] Pauvre monta.i.gnare à la tête trouée de toute part[...]Tant que tout devra se passer par un scrutin, tout camerounais remplissant les conditions d'éligibilité pourra se présenter, indépendamment de son sexe, ses origines, sa religion. ${ }^{10}$

Plus intéressant, l'internaute précise bien dans son post que le jeu démocratique doit être aussi ouvert, y compris quand on est une femme. Celle-ci peut ainsi aspirer jusqu'à la fonction suprême, sujet qui reste encore aujourd'hui tabou dans cette partie du monde. Cette intervention semble en cela répondre à une véritable rationalité démocratique, même si elle est loin de respecter comme observé plus haut toute logique formelle et procédurale (avec notamment l'usage d'un vocabulaire vindicatif et insultant). Il n'est donc pas toujours question de mettre en jeu le moindre processus délibératif, les propos mis en exergue renforçant l'idée d'un espace d'échanges malgré tout lacunaire. Dans le même ordre d'idées, cet autre participant s'inquiète de cette appropriation du pouvoir par la majorité beti en des termes plus frappants :

«Le problème $\mathrm{c}$ que le Betis/Fangs/Boulous sont troooop gates et chanceux. Ils ont tout ce qu'ils veulent chez eux [...] j'esperent qu'ils ne vont pas se facher et chasser tous les immigres de chez eux $»^{11}$ 
24

noint de vue, si d'un côté «majorité » rime avec " peuples autochtones », de l'autre «minorité » relève d'une logique "allogène ». D'ailleurs, il explique que la plupart des grandes infrastructures du Cameroun se retrouvent sur le territoire beti / fang/ boulou. Bien plus, il utilise le terme «d'immigrés » pour nommer la composante allogène de cette population (qui constitue de fait le groupe minoritaire) alors qu'il s'agit manifestement des individus qui sont tous camerounais d'origine. On est en bref dans un cas de figure où le processus d'hégémonisation est tellement poussé que certains individus marginalisés se sentent apatrides. Aussi, l'idée même d'un gouvernement monarchique et autocratique où la figure du président camerounais est associée à un système tribal sans fin est avancée par certains habitués du cyberespace, qui se rangent du côté des catégories marginalisées :

«Le Biyaïsme est un tribalisme. Le futur du Cameroun s'annonce chaotique et Biya s'en fout puisque ' il ne sera plus là. Combien de Fang-beti gouverneur?, préfet, directeur de ceci directeur de cela, c'est un scandale et n'en parlons plus de l'armée $»^{12}$

Il s'agit ici pour l'internaute, d'après nos hypothèses, de tester véritablement ses capacités argumentatives, sans doute en vue d'un engagement dans la vie politique de son pays. Le langage est plus ténu, dans cet exemple. En parlant de «Biyaïsme », en tant que système anti-démocratique et non de "Biya ", en tant que personne de chair et d'os, qui peut subir la loi de l'usure physique, il cherche ainsi à donner plus d'écho à ses propos, dans une sorte d'amplification rhétorique calculée. Un autre internaute va plus loin encore, par rapport à l'intervention précédente, en arguant que le problème de tribalisme et de sous-représentation nationale des minorités au niveau politique dépasse largement les frontières nationales :

«Dans votre intervention du 15-Feb-2015 $04: 21$ EST vous semblez vous faire l'avocat de la propagande mensongere d'Afrovision. J'ai pose a ce dernier des questions bien precises et je vais les repeter. Prenos le cas de l'Ambassade du Cameroun en Allemagne Federale sous le regne de monsieur Paul Biya. Quels sont le nom et l'ethnie de celui qui fut pendant longtemps Ambassadeur du Cameroun dans ce pays ? ${ }^{13}$

Dans cette prise de parole publique, l'internaute se pose en médiateur de la cause des couches qui subissent durement le poids des injustices. De même, il s'agit de profiter de la tribune pour reconfigurer un espace identitaire, habité par tous les oubliés du système. On passe même du stade de légitimité (affirmation comme Camerounais, à part entière), à celui de la reconnaissance (en tant que tel, on doit bénéficier des mêmes droits et devoirs). Plus question, donc, de s'exprimer dans l'unique objectif "d'exister », comme cela a été le cas avec d'autres posts étudiés. Selon l'une des idées avancées par Patrice Flichy (2008), Internet, contrairement aux médias de masse, n'est pas un acteur de ces grands rituels collectifs qui permettent de réaffirmer régulièrement les identités collectives. Il ajoute que la question avait déjà été évoquée par Steven Schneider (1996), qui faisait de la diversité une des trois conditions (avec l'égalité et la réciprocité) d'un espace public habermassien sur Internet. C'est effectivement ce qui se donne à voir dans certaines occurrences étudiées : il n'est pas toujours évident de dire quel groupe relève des minorités ou pas. C'est pour cela que l'hypothèse finale de "minorités complexes ", parlant du cas du Cameroun, n'est pas une réalité dénuée de fondement. 


\section{Conclusion}

$27 \mathrm{Au}$ terme de notre analyse, quels liens établir finalement entre Internet, réseaux sociaux et démocratie? Le cyberespace camerounais est-il un lieu de balkanisation de l'espace public, de désinformation, de morcellement de l'opinion, d'émiettement de l'esprit de communauté, de colportage de rumeur, de communautarisation de l'opinion, bref de « fracture démocratique " ? S'il n'est pas aisé de répondre de façon appropriée à ces différentes interrogations, il faut quand même reconnaître que les interactions communicationnelles en ligne sont un vrai moment d'appropriation de la parole surtout par tous ceux qui n'ont pas droit au chapitre. Nouvel environnement d'engagement politique, Internet constitue en somme un outil adapté aux nouvelles formes de militantisme, un haut lieu d'où émerge une sorte de démocratie réticulaire pour parler comme Fabien Granjon (2011).

\section{BIBLIOGRAPHIE}

Bibliographie

Boughzala Younès, Inès Bouzid et Moscarola Jean, « Le rôle des réseaux sociaux et des TIC dans les révolutions arabes : les résultats d'une enquête » [en ligne] http://www.lesphinxdeveloppement.fr/blog/wp-content/uploads/2012/04/YB_IB_JM1.pdf (site visité le 10 juillet 2015).

Castells Manuel, La galaxie Internet, Fayard, Paris, 2001.

Crozier Michel, Friedberg Ehrard, L'acteur et le système. Les contraintes de l'action collective, Seuil, Paris, 1981.

Dumoulin Marc, « Les forums électroniques : délibératifs et démocratiques? », in D. Monière, (dir.), Internet et la démocratie, Monière et Wollank, Québec, 2002, p. 141-157.

Flichy Patrice, « L'individualisme connecté, entre la technique numérique et la société », Réseaux, vol. 22, n 124, 2004, p. 17-51.

Flichy Patrice, « Internet et le débat démocratique », in Greffet, F., et Wojcik, S., (dir.), Parler politique en ligne, Réseaux, 2008/4 n 150, 2008, p. 159-185. DOI : 10.3917/res.150.0159

Freitag Michel, « De la terreur au meilleur des mondes. Globalisation et américanisation du monde : vers un totalitarisme systémique», in Dagenals, D., (dir.), Hannah Arendt, le totalitarisme et le monde contemporain, Presses de l'Université Laval, Québec, 2003.

Grallet Guillaume, Mandonnet Eric, Karlin Elise, Rosso Romain, « Internet : Enquête sur le cinquième pouvoir » [en ligne] http://lexpansion.lexpress.fr/high-tech/enquete-sur-lecinquieme-pouvoir_479170.html (site visité le 27 janvier 2015)

Granjon Fabien, « Mouvement anti-mondialisation et dispositifs de communication sur réseaux », Colloque Bogues, 2001, Globalisme et pluralisme, Presses de l'université de Laval, Laval, Tome 4, 2001, p. 137-154. 
Habermas Jürgen, L'espace public : Archéologie de la publicité comme dimension constitutive de la société bourgeoise, Payot, Paris, 1993.

Konings Piet, Nyamnjoh Francis, " The Anglophone Problem in Cameroon », The Journal of Modern African Studies, Vol. 35, No. 2, 1997, p. 207-229.

Licklider Joseph, Taylor Robert, « The Computer as a Communication Device », Science and Technology, April 1968, reprinted in Memoriam :J.C.R. Licklider 1915-1990, Digital Systems Research Center, Palo Alto, California, 1990.

Morozov Evgeny, Pour tout résoudre, cliquez ici, L'aberration du solutionnisme technologique, Fyp, Limoges, 2014.

Mouangue Bonam Kobila Gabriel James, La protection des minorités et des peuples autochtones au Cameroun. Entre reconnaissance interne contrastée et consécration universelle réaffirmée, Dianoïa, Paris, 2009.

Mouiche Ibrahim, Démocratisation et intégration sociopolitique des minorités ethniques au Cameroun: entre dogmatisme du principe majoritaire et centralité des partis politiques, CODESRIA, Dakar, 2010.

Ngando Sandjé Rodrigue, «Le droit des minorités et des peuples autochtones au Cameroun » : une lecture actuelle et éventuelle », Droit et cultures [en ligne], 66 | 2013-2, mis en ligne le 14 janvier 2014. http://droitcultures.revues.org/3256 (site visité le 04 mars 2015)

Njoya Jean, «États, peuples et minorités en Afrique Sub-saharienne », Janus, vol. 5, n³, 2011. Pelican Michaela, « Mbororo claims to regional citizenship and minority status in northwest Cameroon », Africa 78 (4), 2008, p. 540-560.

Pisier Évelyne, Varikas, Eleni, « Femmes, République et démocratie. L'autre dans la paire ? », Pouvoirs, n 82, 1997, p. 127-143.

Rouland Norbert, « Anthropologie juridique », Définir le droit 2, Droits, 1990, nº 11.

Salmon Jean, (dir.), Le dictionnaire de droit international public, Bruxelles, Bruyland/ AUF, coll. «Universités francophones », 2001.

Schneider Steven, «Creating a Democratic Sphere Through Political Discussion », Social Science Computer Review, vol. 14, n 4, 1996, p. 373-393.

Stenger Thomas, «La prescription de l'action collective » Double stratégie d'exploitation de la participation sur les réseaux socionumériques, Hermès, La Revue, 2011/1 n 59, 2011, p. 127-133. Sumpf Joseph, Hugues Michel, Dictionnaire de sociologie, Larousse, Paris, 1973.

Trippi Joe, The Revolution Will Not Be Televised: Democracy, the Internet and the Overthrow of Everything, Harper Collins, New York, 2008.

http://www.cameroon-info.net/

http://www.camer.be/

\section{NOTES}

\section{1. http://www.cameroon-info.net/}

2. http://www.camer.be/

3. Ce rapport a été transmis à l'Agence France Presse et les grandes lignes sont disponibles sur le site du monde dans sa livraison du 17.10.2012: http:// 
www.lemonde.fr/afrique/article/2012/10/17/la-liberte-d-expression-est-en-dangerau-cameroun_1776464_3212.html\#CVQGDIXsSsDwsLaM.99

4. L'ensemble des lois, textes réglementaires et décrets relatifs aux télécommunications et aux nouvelles technologies numériques peuvent être consultés sur les sites : http:// www.art.cm/index.php/fr/art-actu \& http://www.antic.cm/

5. http://www.lemonde.fr/afrique/article/2015/01/26/achille-mbembe-venez-enafrique-venez-chez nous_4563211_3212.html\#2xutvcJ6fFPOQ3lq.99

6. http://www.camer.be/24315/6:1/camerounbassek-ba-kobhio-lapres-biya-minquietejai-peur-de-livoirisation-du-cameroun-cameroon.html

7. http://www.cameroon-info.net/reactions/@,65328,7,cameroun-paul-biya-a-celebrece-jour-sans-tambour-ni-trompette-son-82e-anniversa.html

8. http://www.camer.be/24315/6:1/camerounbassek-ba-kobhio-lapres-biya-minquietejai-peur-de-livoirisation-du-cameroun-cameroon.html

9. http://www.camer.be/24315/6:1/camerounbassek-ba-kobhio-lapres-biya-minquietejai-peur-de-livoirisation-du-cameroun-cameroon.html

10. http://www.cameroon-info.net/reactions/@,65328,7,cameroun-paul-biya-acelebre-ce-jour-sans-tambour-ni-trompette-son-82e-anniversa.html

11. http://www.cameroon-info.net/reactions/@,65328,7,cameroun-paul-biya-acelebre-ce-jour-sans-tambour-ni-trompette-son-82e-anniversa.html

12. http://www.cameroon-info.net/reactions/@,65328,7,cameroun-paul-biya-acelebre-ce-jour-sans-tambour-ni-trompette-son-82e-anniversa.html

13. http://www.cameroon-info.net/reactions/@,65328,7,cameroun-paul-biya-acelebre-ce-jour-sans-tambour-ni-trompette-son-82e-anniversa.html

\section{RÉSUMÉS}

L'accès des minorités (ethniques) et des entités marginalisées (femmes, jeunes) au pouvoir démocratique au Cameroun via les réseaux et médias socionumériques connaît un tournant majeur avec la prolifération des nouvelles technologies de l'information et de la communication. $\mathrm{Au}$ travers de deux plateformes camerounaises de discussion en ligne, à savoir Camer be et Cameroun.info-net, nous montrons qu'elles constituent désormais un vecteur d'émancipation qui permet à ces entités sociales longtemps muselées par les différents pouvoirs en place de reprendre l'initiative en matière de liberté d'expression et de culture démocratique. Dès lors, Internet assure non seulement une grande visibilité sociopolitique malgré la chape de plomb de la censure qui demeure menaçante pour ces usagers singuliers du web, mieux ils profitent de ces moments d'échanges interactifs pour éprouver leur compétence communicationnelle, même si celle-ci est parfois limitée, sinon embryonnaire. L'idée d'une démocratie participative impulsée par les groupes étudiés devient ainsi une réalité en marche, le cyberespace camerounais se dotant de plus en plus d'un pouvoir de libérer et fédérer, ce qui leur donne la possibilité de participer pleinement au grand débat national sur des questions touchant aussi bien le politique, l'économique, le social que le culturel. 
The access of (ethnic) minorities and marginalized entities (women, young people) to the democratic power in Cameroon through socio-numeric networks and media is experiencing a major turning point with the proliferation of new information and communication technologies. Through two Cameroonian platforms of online discussion, namely Camer be and Cameroun.infonet, I show that they constitute from now on a vector of empowerment that allows these social entities for a long time muzzled by the various powers in place to take back the initiative regarding freedom of expression and democratic culture. Therefore, Internet not only insures a great sociopolitical visibility despite the leaden weight of censorship that remains a threat to these singular web users, better they take advantage of these moments of interactive exchanges to test their communicational skills, even if it is sometimes limited, otherwise embryonic. The idea of a participative democracy driven by the studied groups so becomes a reality on the move, the Cameroonian cyberspace acquiring more and more power to liberate and unite, what gives them the opportunity to fully participate in the great national debate on issues affecting as well as the political, the economic, the social as the cultural.

\section{INDEX}

Keywords : Social networks, minorities, communication, democracy, Internet Mots-clés : Réseaux sociaux, minorités, communication, démocratie, Internet

\section{AUTEURS}

\section{ABOUBAKAR SIDI NJUTAPWOUI}

Université de Yaoundé 2

\section{JEAN PIERRE FEWOU NGOULOURE}

Université de Toulouse 2, CPST-LERASS

Fr 